\title{
COMPORTAMENTO DA CAREPA TERCIÁRIA NO ARCO DE CONTATO NA LAMINAÇÃO DE TIRAS A QUENTE *
}

\author{
Antonio Adel dos Santos ${ }^{1}$ \\ Arthur de Castro Souza ${ }^{2}$ \\ Geraldo Arruda Maia ${ }^{3}$
}

\begin{abstract}
Resumo
Visando elucidar o comportamento da carepa terciária durante a laminação de tiras a quente, em um aço produzido como laminado a quente e com rigorosa exigência de qualidade superficial, foi realizada uma parada forçada do laminador, impondo-se, imediatamente, um resfriamento rápido da superfície da tira. Amostras coletadas na região do arco de contato mostraram que a carepa possui espessura na faixa de $10 \mu \mathrm{m}$ a $14 \mu \mathrm{m}$ na entrada das cadeiras, sendo reduzida para $7 \mu \mathrm{m}$ com a deformação, sem ocorrência de ruptura. Tais características indicam que, do ponto de vista do comportamento no trem acabador, a carepa apresenta espessura e plasticidade adequadas para evitar defeitos nos produtos laminados. A carepa foi constituída predominantemente por $\mathrm{Fe}_{3} \mathrm{O}_{4}$, óxido plástico na temperatura de laminação. Foi desenvolvido e aplicado um modelo de crescimento da carepa, que forneceu valores de espessura muito próximos dos medidos, se tornando uma ferramenta útil para análise da influência de condições de processo na carepa.
\end{abstract}

Palavras-chave: Laminação a quente; Carepa terciária; Defeitos superficiais.

\section{BEHAVIOR OF TERTIARY SCALE IN THE ARC OF CONTACT DURING HOT STRIP ROLLING}

\begin{abstract}
In order to elucidate the behavior of tertiary scale during hot strip rolling of steel with tight surface quality requirement, a forced stop of the finishing mill was done, followed by rapid cooling of the strip surface. Samples collected in the arc of contact have shown scale thickness in the range from $10 \mu \mathrm{m}$ to $14 \mu \mathrm{m}$ at each stand entry, being reduced to about $7 \mu \mathrm{m}$, concomitant with the deformation, without ruptures. This means that its thickness and plasticity are suitable for rendering good surface quality in the end rolled product. The scale was composed mainly by $\mathrm{Fe}_{3} \mathrm{O}_{4}$, which is an oxide with enough plasticity to be deformed in the rolling temperature. In addition, it was developed a prediction model for the scale growth, which provided results of scale thickness very close to the experimental values. This model can be a useful tool for investigating the influence of processing variables on scale thickness.
\end{abstract}

Keywords: Hot rolling; Tertiary scale; Surface defects.

1 Engenheiro Metalurgista, DSc., CQE/ASQ, Pesquisador Especialista Sênior, Centro de Pesquisa, Usiminas, Ipatinga, MG, Brasil.

2 Engenheiro Metalurgista, CQE/ASQ, Engenheiro de Processos da Laminação a Quente, Usiminas, Ipatinga, MG, Brasil ‘

3 Engenheiro Metalurgista, CQE/ASQ, Gerente-Geral da Laminação a Quente, Usiminas, Ipatinga, MG, Brasil.. 


\section{INTRODUÇÃO}

A formação de uma camada de óxidos, chamada carepa, na superfície do aço durante a laminação a quente é inevitável, em função da alta afinidade do Fe com o $\mathrm{O}$, das elevadas temperaturas do processo e da presença de agentes oxidantes no meio, por exemplo, $\mathrm{O}_{2}, \mathrm{H}_{2} \mathrm{O}$ e $\mathrm{CO}_{2}$. Existe uma vasta literatura sobre a oxidação do ferro puro e de aços em temperaturas elevadas, de forma que o mecanismo de formação da carepa, em geral, é bem conhecido $[1,2,3]$.

Desta oxidação superficial decorrem várias implicações tecnológicas na produção dos aços, tais como: (i) perda metálica afetando negativamente o rendimento do processo de laminação; (ii) geração de defeitos nos produtos laminados; (iii) desgaste dos cilindros de laminação e efeito nas cargas de laminação; (iv) influência em processos subsequentes de conformação e de decapagem do produto laminado. A carepa terciária é aquela formada após a última descarepação, que, numa linha convencional de tiras a quente, é normalmente na entrada do trem acabador. Esta carepa permanece sobre $\mathrm{O}$ aço até o processo de decapagem da bobina. A presença recorrente de defeitos superficiais em bobinas a quente, como laminadas ou como decapadas, advindos da carepa terciária, têm motivado diversos estudos fundamentais $[4,5,6]$ visando entender seu comportamento durante a laminação e relacioná-lo com os defeitos. Idealmente, a carepa terciária deve ser fina, uniforme e com plasticidade suficiente para ser deformada junto com o metal durante os passes [7].

A maioria dos estudos tem sido realizada em laboratório, onde, por mais que se tente aproximar das condições reais da laminação industrial, ainda ficam desconsiderados vários efeitos de difícil simulação. Portanto, é preciso ser muito criterioso para a transposição desses resultados laboratoriais para o processo industrial. Também é muito temerária a aplicação dos resultados obtidos em uma linha industrial para outra, uma vez que a carepa depende da configuração da linha e de vários parâmetros específicos, tais como distância e quantidade de laminadores, resfriamentos aplicados ao esboço e velocidades de processo.

Neste sentido, o objetivo deste trabalho é contribuir para o entendimento do comportamento da carepa terciária no processo de laminação de tiras a quente. Para isso, foi realizada uma parada forçada do laminador e retiradas amostras da tira na região do arco de contato de cada cadeira, as quais foram caracterizadas em detalhes. Também foi desenvolvido um modelo de previsão da espessura da carepa, validado pelas determinações de espessura medida nas amostras.

\section{METODOLOGIA}

\subsection{Parada forçada do trem acabador}

Foi realizada a parada forçada do trem acabador durante a laminação de uma tira de aço $\mathrm{CMnNb}$, cuja aplicação exige excelente qualidade superficial. A parada foi executada fechando-se as guardas e imobilizando a tira. Imediatamente, o cilindro de trabalho superior de cada cadeira foi levantado para evitar a geração de trincas térmicas em sua superfície devido ao contato com a tira, e foram acionados os resfriamentos entre cadeiras para resfriar a superfície da tira o mais rapidamente possível. Com isso, a composição de fases e a espessura da carepa puderam ser preservadas para as análises. Na figura 1 é mostrada uma fotografia da tira no trem, obtida logo após a parada, no início do resfriamento. 

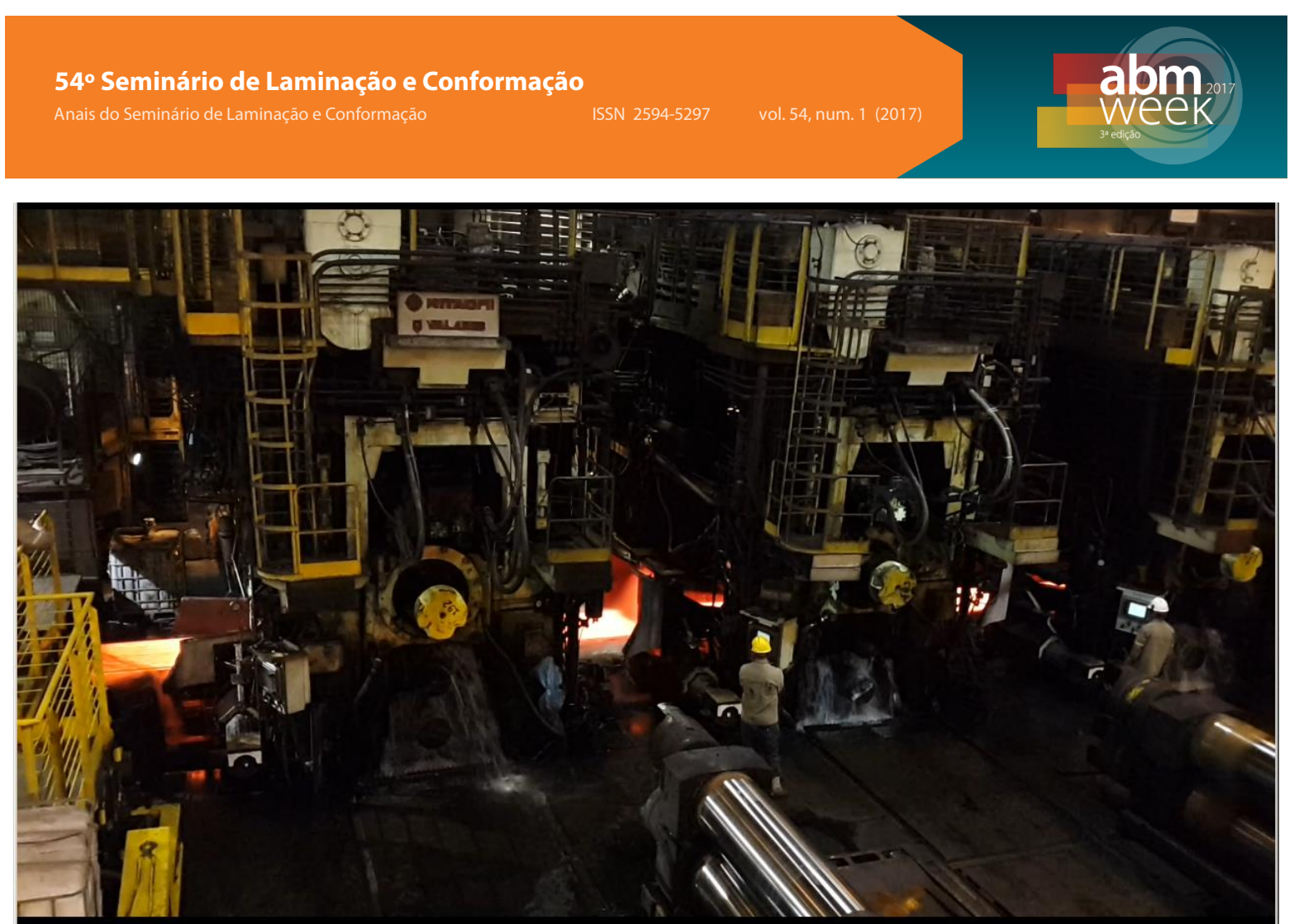

Figura 1. Fotografia da tira nas primeiras cadeiras do trem acabador, logo após a parada.

Após a remoção da tira sucateada, foram cortadas amostras em cinco posições ao longo da largura, na região do arco de contato, para todas as seis cadeiras, contabilizando trinta amostras. Cada uma dessas foi dividida longitudinalmente em várias subamostras, para serem analisadas por diferentes técnicas.

\subsection{Análises realizadas}

a) Medição do perfil da tira no arco de contato

Foram feitas medições da espessura da tira na região do arco de contato e determinada a redução a frio, comparando-a com o cálculo do modelo de set up.

b) Caracterização da carepa por microscopia ótica - MO

Na figura 2 é mostrado, como exemplo, o esquema de posição das análises da carepa em cada subamostra destinada à MO. A região A é logo antes do arco de contato, a região I, varre o arco de contato, e a $\mathrm{D}$, logo depois. Seções longitudinais nas regiões $A$, I e D, preparadas por procedimentos padrão de metalografia, foram observadas ao microscópio ótico, na condição como polida e como atacada pelo reagente composto por uma solução de ácido clorídrico em etanol a 3\% (cloral). Este ataque visa destacar os óxidos constituintes da carepa.

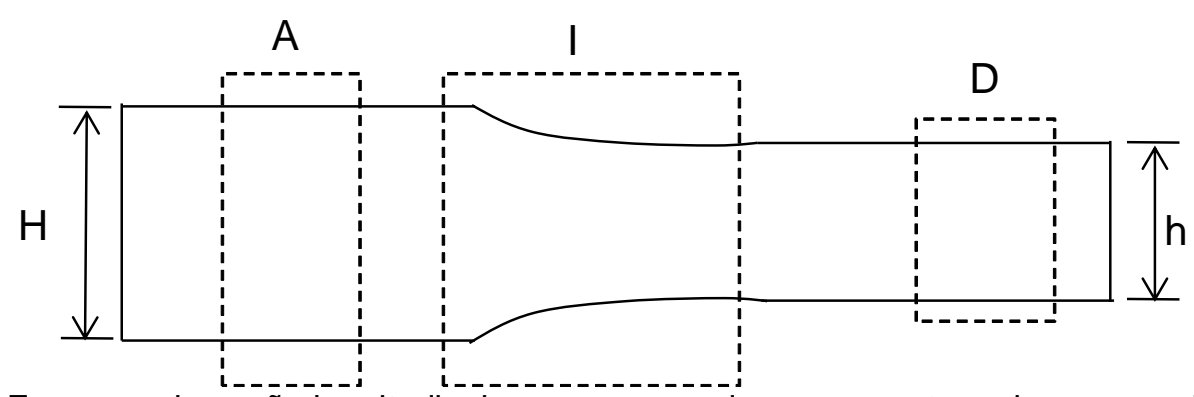

Figura 2. Esquema da seção longitudinal na espessura de uma amostra sobre o arco de contato, destacando os locais de análises (retângulos tracejados). H: espessura de entrada; h: espessura de saída. 


\section{c) Análise por MEV/INCA}

Seções observadas ao MO das amostras de todas as cadeiras foram analisadas por microscopia eletrônica de varredura (MEV), em equipamento Zeiss Evo 50, usando análises quantitativas por EDS e o software INCA, Oxford, com tensão de $10 \mathrm{kV}$. O objetivo foi determinar a composição química em vários locais da carepa e definir os seus óxidos constituintes.

d) Análise por GDOES (glow discharge optical spectroscopy)

A análise por GDOES permite determinar o perfil de concentração dos elementos químicos a partir da superfície da amostra. Algumas amostras foram submetidas à análise por esta técnica com o objetivo de verificar a segregação dos elementos químicos na interface metal/carepa e determinar a espessura da camada de carepa. O equipamento usado foi o LECO GDS 850A.

e) Difratometria de raios- $X(D R X)$

Foram realizadas análises de superfície para determinação dos óxidos presentes na carepa, calculando-se sua proporção pela técnica de Rietveld. O equipamento usado foi Bruker Advance D8, operando com $40 \mathrm{kV}$.

\subsection{Modelo crescimento da carepa}

$\mathrm{Na}$ figura 3 é mostrado o esquema da Linha de Tiras a Quente, em Ipatinga, destacando-se a região de aplicação do modelo de crescimento de carepa, pelo retângulo vermelho, desde a entrada da tesoura de pontas (crop shear) até a saída do trem acabador, na posição do pirômetro.

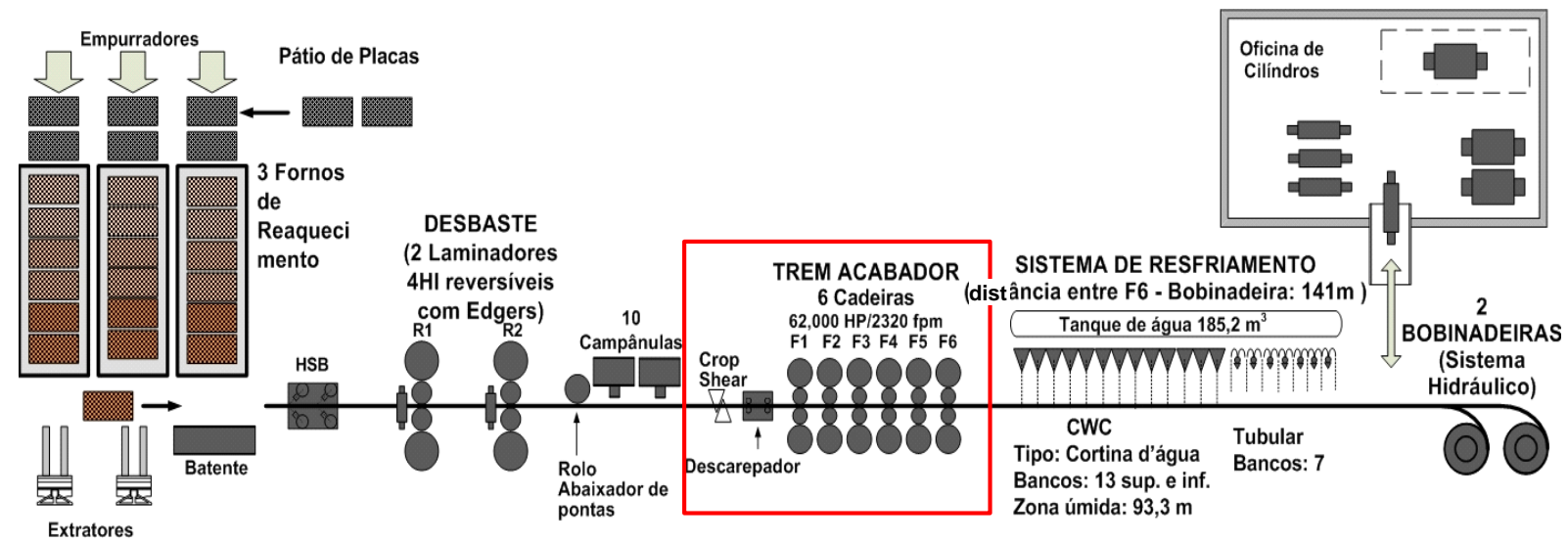

Figura 3. Esquema da linha de tiras a quente em Ipatinga, destacando a região de laminação de acabamento.

Para prever o crescimento da carepa é necessário conhecer a evolução térmica da tira durante a passagem no trem acabador. Para isso foi adaptado o modelo on-line de controle do processo, fornecido pela Converteam, cujo núcleo é a equação fundamental de transferência de calor unidimensional, dada em (1). Aplicando-se as condições inicial e de contorno, esta equação é resolvida numericamente por método implícito de diferenças finitas.

$$
\rho C[T(t, x)] \frac{\partial T(t, x)}{\partial t}=\frac{\partial}{\partial x}\left\{k[T(x, t)] \cdot \frac{\partial T(t, x)}{\partial x}\right\}
$$


$\mathrm{T}$ : temperatura $(\mathrm{K})$;

$\rho$ : densidade do aço $\left(\mathrm{kg} \cdot \mathrm{m}^{-3}\right)$;

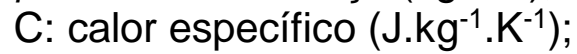

$\mathrm{k}$ : condutividade térmica $\left(\mathrm{W} \cdot \mathrm{m}^{-1} \cdot \mathrm{K}^{-1}\right)$;

$\mathrm{x}$ : posição ao longo da espessura do laminado $(\mathrm{m})$;

$\mathrm{t}$ : tempo decorrido (s).

Para conceber o modelo de crescimento da carepa, é preciso ter em mente que ela é constituída por três óxidos, $\mathrm{FeO}, \mathrm{Fe}_{3} \mathrm{O}_{4}$ e $\mathrm{Fe}_{2} \mathrm{O}_{3}$. A camada mais interna, de $\mathrm{FeO}$, é formada imediatamente após o contato da superfície com o ar, normalmente obedecendo à cinética linear, dada pela equação (2). Alguns instantes depois, o comportamento parabólico, dado por (3), passa a prevalecer.

$$
\begin{aligned}
& s_{L}=k_{L} t \\
& s_{p}^{2}=k_{p} t
\end{aligned}
$$

s: espessura da camada da carepa $(\mu \mathrm{m})$;

$\mathrm{L}$ e p: subscritos denominando linear e parabólico, respectivamente;

$\mathrm{t}$ : tempo de oxidação (s);

$\mathrm{k}_{\mathrm{p}}$ : constante parabólica de oxidação isotérmica $\left(\mu \mathrm{m}^{2} \cdot \mathrm{s}^{-1}\right)$;

$\mathrm{kL}$ : constante linear de oxidação isotérmica $\left(\mu \mathrm{m} \cdot \mathrm{s}^{-1}\right)$.

Foi adotado um modelo misto em que a cinética linear prevalece até um instante crítico, tc, determinado pela equação (4), quando a cinética passa a obedecer ao modelo parabólico. A figura 4 ilustra essa concepção.

$$
t_{c}=\frac{k_{p}}{2 k_{L}^{2}}
$$

Existe uma diversidade de equações de cinética de oxidação do ferro puro e dos aços. Algumas delas fornecem resultados muito parecidos. Então foram adotados os parâmetros apresentados por Picque [5] para a constante isotérmica de oxidação, equação (5), conforme tabela 1.

$$
k_{(p, L)}=k_{(p, L) 0} \exp \left[-\frac{Q_{(p, L)}}{R T}\right]
$$

$\mathrm{k}_{(\mathrm{p}, \mathrm{L}) 0}$ : coeficiente de oxidação isotérmica $\left(\mu \mathrm{m}^{2} \cdot \mathrm{s}^{-1}\right)$;

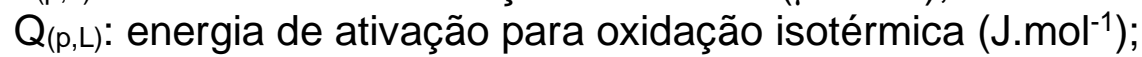

$\mathrm{R}$ : constante universal dos gases $\left(8,314 \mathrm{~J} \cdot \mathrm{mol}^{-1} \cdot \mathrm{K}^{-1}\right)$. 


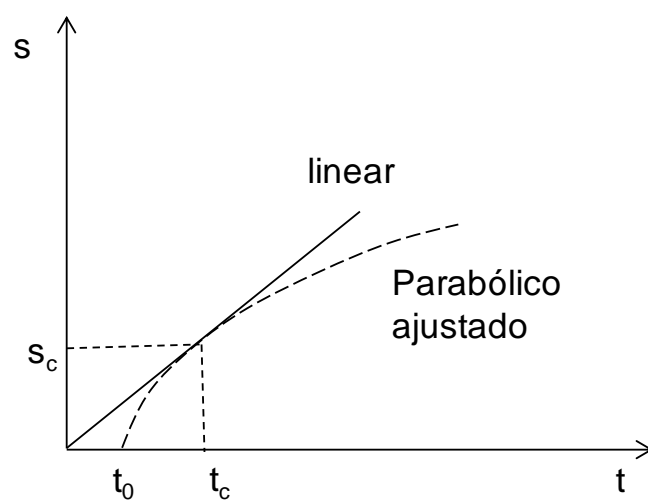

Figura 4. Curvas esquemáticas de crescimento linear e parabólico ajustado para o crescimento isotérmico da carepa.

Todas as equações de cinética de oxidação são para condição isotérmica. Uma vez que a tira passa por resfriamento contínuo, é necessário um procedimento para aplicá-las nessa condição. Optou-se por usar o cálculo do tempo equivalente de espessura da carepa em cada intervalo de temperatura. Os modelos de temperatura e de espessura de carepa foram acoplados em um aplicativo de simulação, que busca dados de processo, dados de configuração da linha, realiza os cálculos e apresenta os resultados numa interface gráfica.

Tabela 1. Constantes da equação (5) usadas no modelo de crescimento de carepa [5].

\begin{tabular}{|c|c|c|c|}
\hline Parâmetro & Unidade & \multicolumn{1}{|c|}{$\mathrm{T}<=1000^{\circ} \mathrm{C}$} & $\mathrm{T}>1000^{\circ} \mathrm{C}$ \\
\hline $\mathbf{k p}_{\mathbf{0}}$ & $\mu \mathrm{m}^{2} . \mathrm{s}^{-1}$ & \multicolumn{2}{|c|}{$5,76 \mathrm{E}+08$} \\
\hline $\mathbf{Q}_{\mathbf{p}}$ & $\mathrm{J} \cdot \mathrm{mol}^{-1}$ & \multicolumn{2}{|c|}{170000} \\
\hline $\mathbf{k L} \mathbf{m}_{0}$ & $\mu \mathrm{m} \cdot \mathrm{s}^{-1}$ & $1,50 \mathrm{E}+04$ & 22,6 \\
\hline $\mathbf{Q L}$ & $\mathrm{J} \cdot \mathrm{mol}-1$ & 89600 & 20300 \\
\hline
\end{tabular}

\section{RESULTADOS E DISCUSSÃO}

\subsection{Perfil da tira no arco de contato}

$\mathrm{Na}$ figura 5 é mostrado, como exemplo, o perfil de espessura da tira no arco de contato da cadeira F1. As mesmas características foram observadas em todas as cadeiras. O gráfico revela que a espessura passa por um valor mínimo no arco de contato, antes de atingir e se estabilizar no valor de saída, formando uma espécie de "poço" de espessura. A maior espessura da tira após o arco de contato, em relação à espessura dentro do arco, pode ser explicada admitindo-se um retorno elástico do material. Destaca-se que este fato não é de conhecimento do autor com base na literatura. A redução a frio medida foi $29,2 \%$, enquanto que o modelo de set up previu $29,4 \%$, portanto muito próximo. 

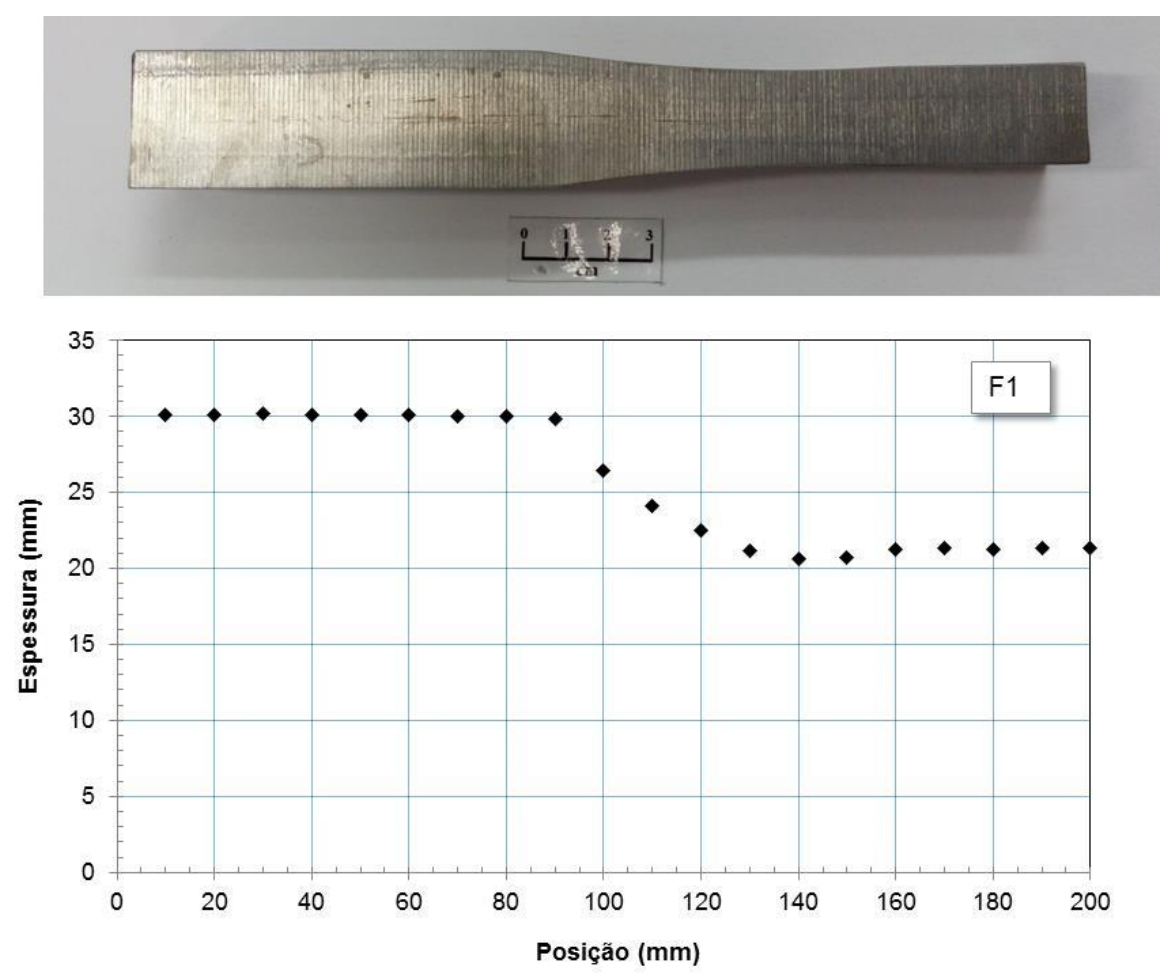

Figura 5. Perfil de espessura da tira na região do arco de contato da cadeira F1.

\subsection{Análise da carepa por MO}

Nas amostras que apresentaram a carepa relativamente íntegra, foi possível examinar seu comportamento no arco de contato. Na figura 6 está mostrada uma sequência de fotografias, como exemplo para a cadeira F1, da camada de carepa desde a mordida até a saída do arco de contato. Não há rompimento da carepa no momento da mordida, e sua espessura é continuamente reduzida até a saída. Localmente na entrada da cadeira, a espessura de carepa varia. No caso da entrada da F1 ela ficou entre 10 e $14 \mu \mathrm{m}$, sendo reduzida para aproximadamente $7 \mu \mathrm{m}$ na saída. Então, a redução plástica da carepa seria entre $30 \%$ e $50 \%$, ou seja, no mínimo igual à redução do metal, de $29 \%$. As análises desta e das amostras de outras cadeiras mostraram que a carepa possui comportamento plástico, e se deforma na mesma proporção ou mais que o metal. Essas observações estão de acordo com indicações da literatura [8]. No entanto, foi verificado que, independente da cadeira e da deformação aplicada, a espessura final da carepa foi em torno de $7 \mu \mathrm{m}$. Esse fato, não comentado na literatura, sugere que há um limite inferior de espessura, abaixo do qual sua plasticidade é restrita.

De acordo com Bolt [7] e Melfo et ali [9], a espessura de carepa não deve ultrapassar $20 \mu \mathrm{m}$ na laminação a quente, além de ser plástica o suficiente para acompanhar a deformação do metal. Assim, a carepa terciária formada no aço em estudo é adequada para a boa qualidade superficial do produto laminado.

$\mathrm{Na}$ maioria das seções analisadas, o ataque com reagente cloral permitiu visualizar duas camadas na carepa, uma interna ocupando cerca de $90 \%$ da espessura, e uma externa de coloração mais clara, figura 7-(a). Este tipo de composição foi praticamente o mesmo em todas as cadeiras. É possível observar que a camada externa recobre algumas trincas verticais. Por ser constituída de uma fase mais oxidada que a camada interna, isto significa que houve penetração de oxigênio por essas trincas e que elas já estavam formadas antes da mordida. Este tipo de trinca na carepa é apresentado na literatura, por exemplo, por Picque [5]. 


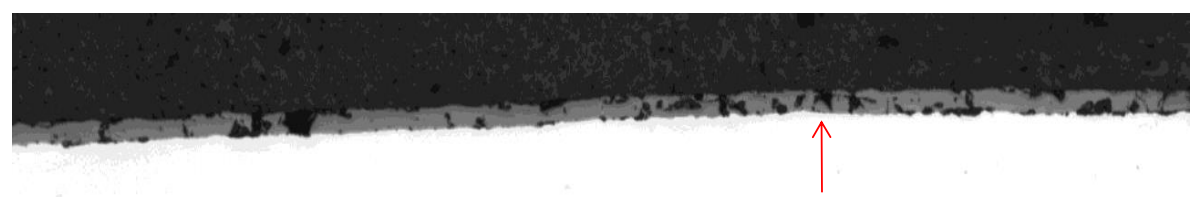

Início do $\mathrm{AC}$

(a) Entrada do arco de contato

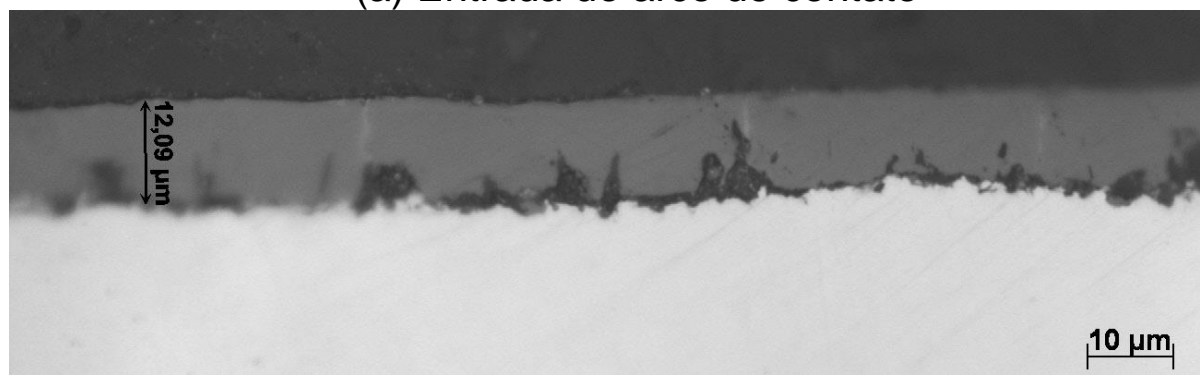

(b) Região inicial dentro do arco de contato

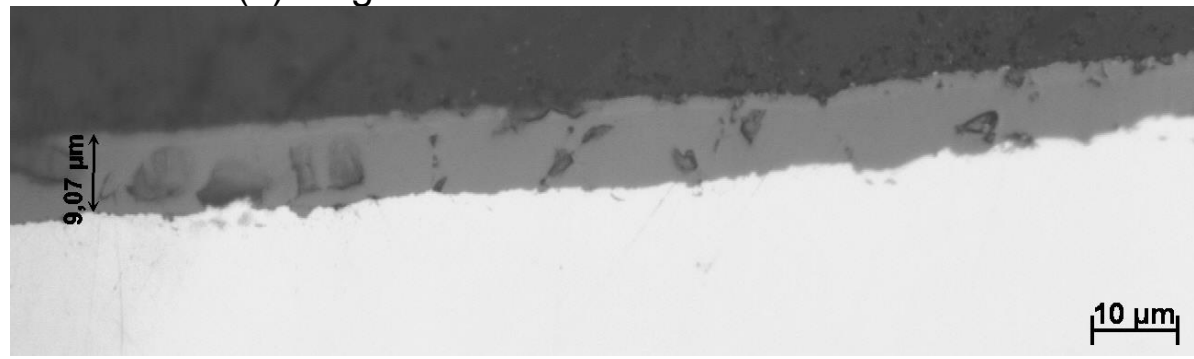

(c) Arco de contato, após (b)

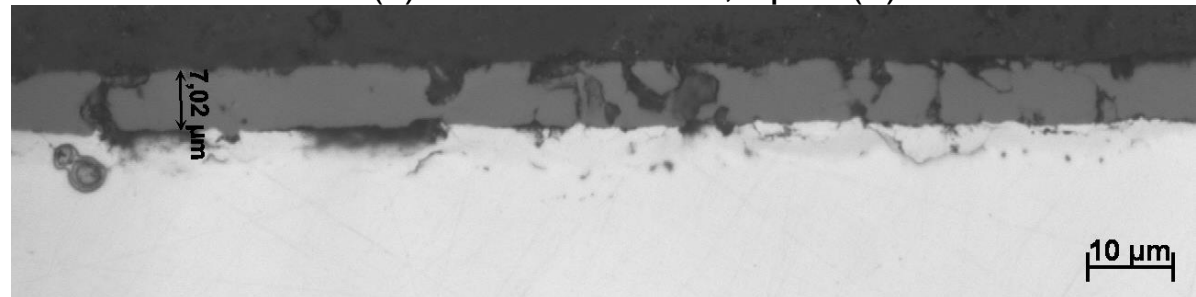

(d) Após o arco de contato

Figura 6. Sequência de fotografias mostrando a evolução da carepa no arco de contato.

Foi visualizada carepa residual da etapa anterior em determinados locais nas amostras da cadeira F1, como mostra a figura 7-(b). A carepa residual ficou sobreposta à carepa terciária, formada a partir da descarepação, e pode resultar em defeitos superficiais no produto. Isso indica que, nas condições de laminação deste aço, cuidado especial precisa ser tomado com a eficiência das descarepações antes da entrada do trem acabador para se garantir a isenção de defeitos no produto laminado.

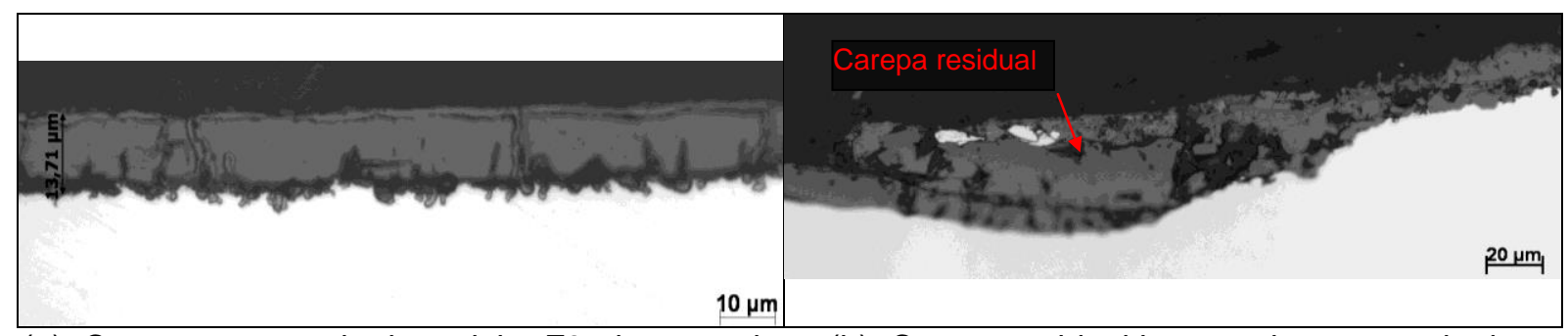

(a) Carepa na entrada da cadeira F3, destacando trincas verticais pré-existentes

(b) Carepa residual incrustada na entrada da cadeira F1

Figura 7. Fotografias de aspectos relevantes encontrados nas análises da carepa ao MO. 


\subsection{Análise por MEV/INCA}

Estas análises indicam os teores de Fe e $\mathrm{O}$, além de outros elementos, nos locais analisados. Foram realizadas várias determinações em várias amostras. $\mathrm{O}$ teor de $\mathrm{Fe}$ estequiométrico do $\mathrm{FeO}, \mathrm{Fe}_{3} \mathrm{O}_{4}$ e $\mathrm{Fe}_{2} \mathrm{O}_{3}$ é, respectivamente, $76 \%, 72 \%$ e $70 \%$. $\mathrm{Na}$ figura 8 é mostrado um exemplo de análise da amostra na entrada da F1. Por comparação com o valor estequiométrico, deduz-se que a camada externa é constituída de $\mathrm{Fe}_{2} \mathrm{O}_{3}$, e a interna, de $\mathrm{Fe}_{3} \mathrm{O}_{4}$.

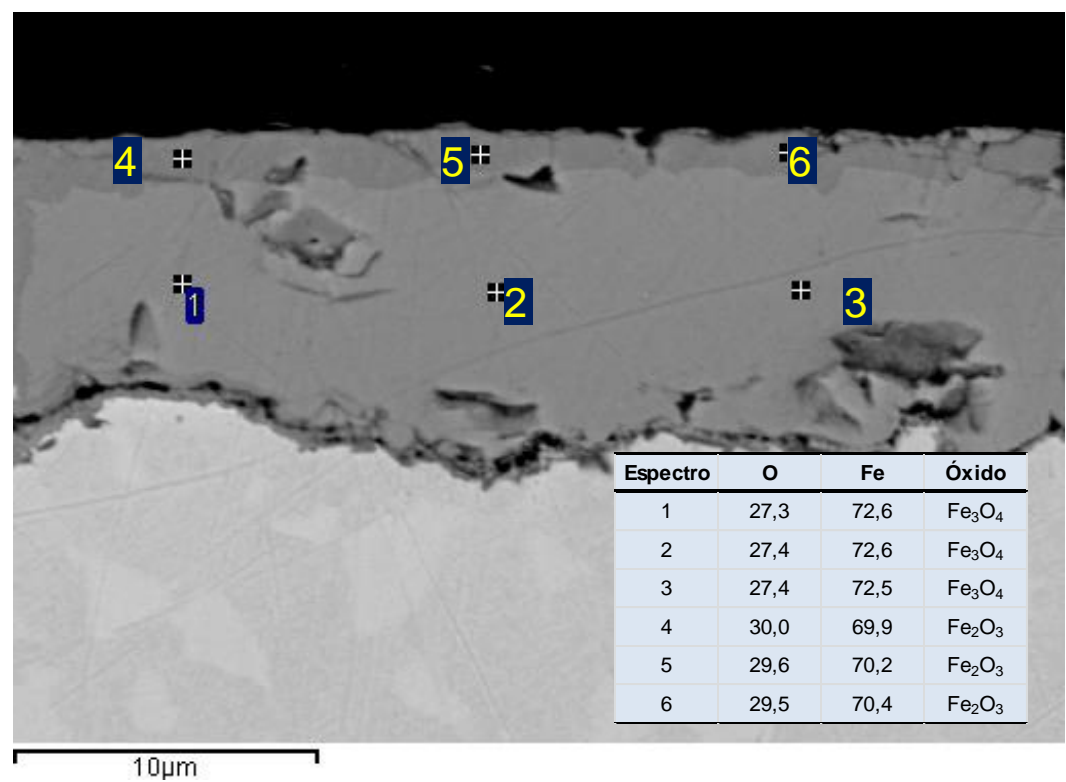

Figura 8. Fotografia de carepa ao MEV na entrada da F1 e análise por MEV/INCA com 10 kV.

\subsection{Análise por GDOES}

Os perfis de concentração dos elementos a partir da superfície da carepa na amostra da cadeira 2, como exemplo, são mostrados na figura 9. Nota-se que o perfil do $\mathrm{Mn}$ tem a mesma tendência daquele do $\mathrm{Fe}$, pois é um elemento substitucional tanto na rede cristalina do $\mathrm{Fe}$ quanto na dos óxidos. Os elementos residuais $P, S$ e Al, segregam na interface carepa/metal, sendo o $P$ mais intensamente. Também elementos presente em traços, que são mais nobres que 0 $\mathrm{Fe}$, quais sejam $\mathrm{Cr}$, $\mathrm{Ni}$ e $\mathrm{Cu}$, ficam enriquecidos na interface. O pico de concentração de tais elementos coincide com o meio da rampa de transição do $\mathrm{Fe} e$ do $\mathrm{O}$, fornecendo uma forma indireta de determinação da espessura da carepa. No caso apresentado a espessura foi de $13 \mu \mathrm{m}$. Quanto aos valores quantitativos de $\mathrm{Fe}$ e O na carepa, os resultados não foram suficientemente precisos, nas análises realizadas, para a distinção dos óxidos presentes. Por exemplo, na figura 9, o teor de Fe ficou próximo de $90 \%$ em toda extensão da carepa, indicando apenas o óxido $\mathrm{Fe}_{2} \mathrm{O}_{3}$, o que foge à realidade. 


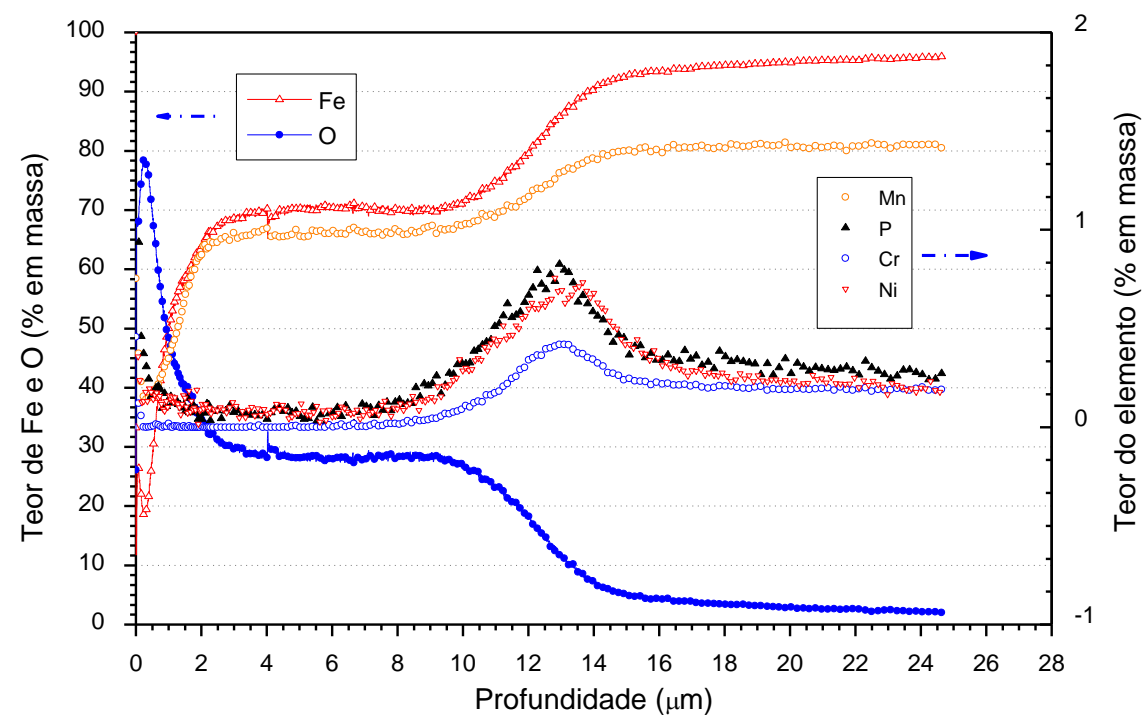

Figura 9. Perfis de concentração dos elementos obtidos por GDOES a partir da superfície da carepa na amostra da cadeira F2. Os teores de P, Cr e Ni estão multiplicados por 10.

\subsection{Análise por DRX}

As análises por DRX indicaram predominância de $\mathrm{Fe}_{3} \mathrm{O}_{4}$ na carepa, pequena quantidade de $\mathrm{Fe}_{2} \mathrm{O}_{3}$, mas também considerável proporção de $\mathrm{FeO}$. A presença de $\mathrm{Fe}_{3} \mathrm{O}_{4}$ e de $\mathrm{Fe}_{2} \mathrm{O}_{3}$ foi confirmada pelas análises por MEV/INCA, como comentado. A presença de FeO, porém, fica difícil de ser explicada. Visualmente ao MO e ao MEV, somente duas camadas foram observadas, que seriam os dois óxidos de maior grau de oxidação. Caso o resfriamento da carepa fosse lento e a camada preponderante de óxido fosse constituída de $\mathrm{FeO}$, poderia haver sua decomposição em $\mathrm{Fe}_{3} \mathrm{O}_{4}$ mais Fe metálico, formando a camada mais espessa interna. No entanto, a superfície da tira foi resfriada muito rapidamente, o que torna altamente improvável tal reação, que ocorre tipicamente após o bobinamento [3]. Além disso, não foram observadas partículas metálicas no interior da camada interna. De acordo com o diagrama apresentado por Min et alli [10], figura 10, na faixa de temperatura de laminação usada, esperar-se-ia uma carepa formada pelos três óxidos, de acordo com os resultados de DRX. Portanto, a presença de $\mathrm{FeO}$ no resultado nesta análise ainda não ficou esclarecida.

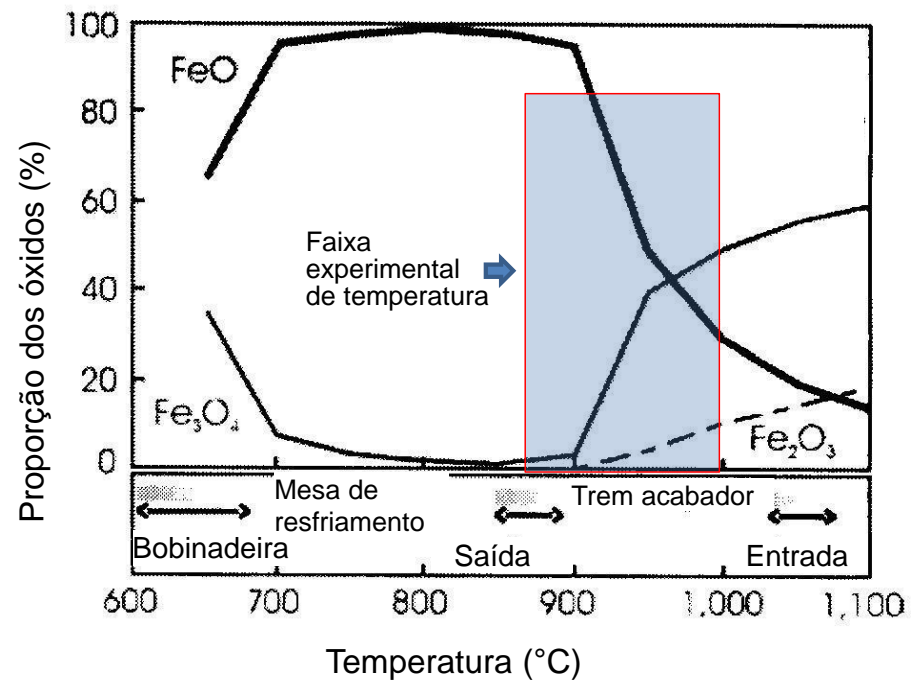

Figura 10. Diagrama de composição dos óxidos da carepa [10], indicando a faixa de temperatura superficial da tira na experiência realizada. 


\subsection{Aplicação do modelo de previsão de espessura}

$\mathrm{Na}$ figura 11 é mostrado o gráfico com o perfil de espessura de carepa calculado com o modelo desenvolvido, além dos valores experimentais determinados com o auxílio das técnicas de MO e de GDOES. Também são mostrados os perfis de temperatura da superfície, Ts, e do centro da tira,Tc, calculados com o modelo para as condições de processo da experiência. Nota-se uma ótima aproximação entre valores medidos e calculados, especialmente para as três primeiras cadeiras de laminação e para a espessura de saída nas cadeiras. Sabe-se que a espessura de carepa, e também os defeitos por origem em carepa, dependem muito da temperatura de processamento.

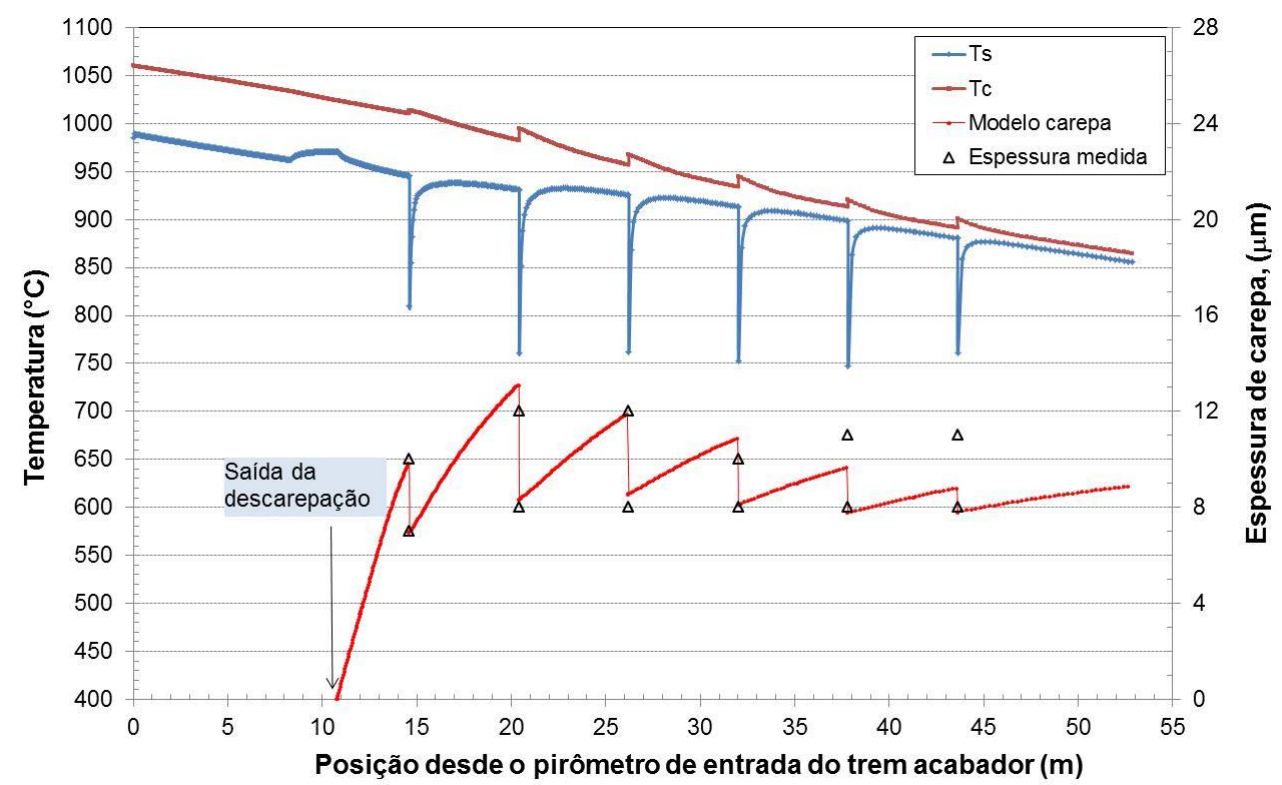

Figura 11. Curvas calculadas com o modelo desenvolvido para a temperatura e a espessura da carepa, esta comparada com valores experimentais.

O modelo desenvolvido permite verificar não só o efeito da temperatura, mas também do tempo de processo, de forma acoplada, na espessura da carepa. Dado que uma excessiva espessura pode levar a problemas de qualidade superficial por carepa, o modelo tem, portanto, o potencial de subsidiar as análises de eventuais ocorrências. Na figura 12 é mostrado o perfil de espessura calculada da carepa ao longo do trem acabador para um conjunto de bobinas laminadas, que não tiveram nenhuma ocorrência de defeito. Nota-se que a espessura não ultrapassa $12 \mu \mathrm{m}$.

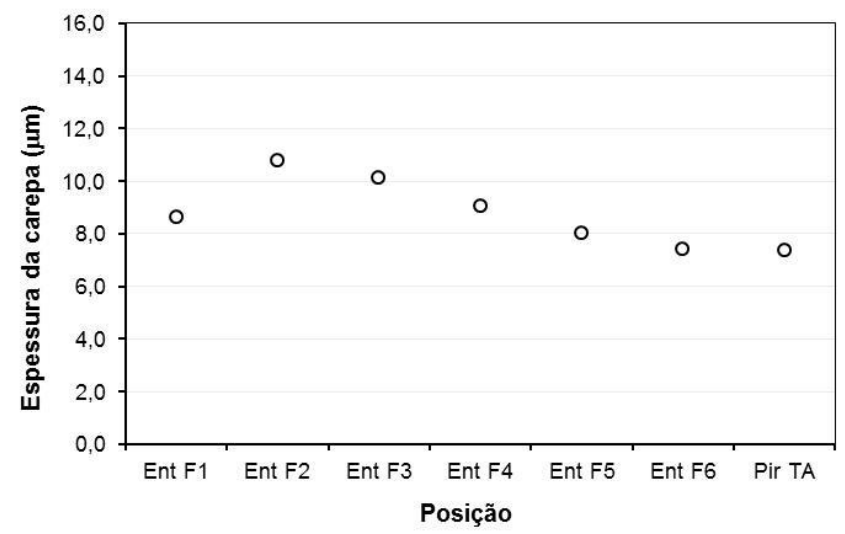

Figura 12. Espessura da carepa calculada na entrada de cada cadeira na posição do pirômetro de saída (Pir TA) do trem acabador para bobinas isentas de defeito. 


\section{CONCLUSÃO}

A carepa terciária no aço estudado, que possui alta exigência de qualidade superficial, apresentou espessura próxima de 10 a $14 \mu \mathrm{m}$ na entrada do arco de contato, portanto, dentro do limite de $20 \mu \mathrm{m}$, considerado adequado para evitar problemas de qualidade no produto.

Esta carepa possui plasticidade suficiente para acompanhar a deformação aplicada no passe de laminação, sendo reduzida até a espessura de $7 \mu \mathrm{m}$, ou seja, no mínimo igual à deformação do metal.

Cuidado especial deve ser tomado com as descarepações antes da entrada do trem acabador, pois eventual presença de carepa residual pode gerar problemas de qualidade superficial, pois causam espessura local da carepa acima de $20 \mu \mathrm{m}$ e incrustam no metal.

Não foi possível determinar precisamente a composição da carepa em termos de porcentagem de óxidos, devido à divergência entre as várias técnicas usadas, mas ficou claro que ela é constituída predominantemente de $\mathrm{Fe}_{3} \mathrm{O}_{4}$, que é um óxido de plasticidade elevada na temperatura de laminação.

O modelo de previsão de crescimento da carepa terciária forneceu resultados bem próximos dos valores medidos de espessura da carepa ao longo do laminador, constituindo-se, portanto, em ferramenta útil para avaliar o efeito de condições de laminação.

\section{REFERÊNCIAS}

1 Tuck CW, Barlow J. The effect of reheating furnace atmosphere on the adhesion of scale to steel. Iron and Steel. 1972; February: 31-38.

2 Sheasby JS, Boggs WE, Turkdogan ET. Scale growth on steels at $1200^{\circ} \mathrm{C}$ : ratinonale or rate and morphology. Metals Science. 1984; March v.18:127-136.

3 Chen RY, Yuen WYD. Review of high temperature oxidation of iron and carbon steels in air or oxygen. Oxidation of Metals. 2003;59(5/6): 433-468.

4 Picard M, Van Den Eynde M, Zachetti N, Kömi J, Lehtinien M. Control of surface layer for pickle-free hot-rolled strip. Final report EUR 22852, European Comission, 2008.

5 Picque B. Experimental study and numerical simulation of iron oxide scales mechanical behavior in hot rolling. These Docteur, l'Ecole de Mines de Paris, 2004, September.

6 Graf M, Kawalla R. Scale development on steel during hot strip rolling. In: Proceedings Rolling 2013: 9th International and 6th European Conference, 2013, Venice, Italy.

7 Bolt PH. Understanding the properties of oxide scales on hot rolled steel strip. Steel Research International. 2004;75(6): 399-404.

8 Krzyzanowski M, Beynon JH, Ferrugia DCJ. Oxide scale behavior in high temperature metal processing. Wiley-VCH, Darmstadt, Alemanha, 2010.

9 Melfo WM, Dippenaar R.J, Reid MH. In-situ study of scale formation under finishing-mill operating conditions. In: AISTech Proceedings, 2006;v. II, p. 25-35.

10 Min K, Kim K, Kim SK, Lee DJ. Effects of oxide surface defects during hot rolling processes. Met. Mat. Inter., Springer, 2012;18(2):341-348. 